\title{
FRAMMENTAZIONE, POLARIZZAZIONE E CLEAVAGES: DEMOCRAZIE FACILI E DIFFICILI
}

\author{
di Giacomo Sani e Giovanni Sartori
}

Lo scopo di questo saggio è di esaminare alcune caratteristiche degli elettorati di otto nazioni. In particolare ci proponiamo di mettere in luce le similarità e differenze tra i sistemi partitici di questi paesi sotto il profilo della frammentazione, polarizzazione, e delle strutture delle linee di divisione (cleavages). Riteniamo che un'analisi basata su queste variabili consenta di mettere a fuoco con maggior precisione il problema del buono o cattivo funzionamento dei sistemi democratici. Riteniamo anche che il ricorso all'analisi comparata possa arricchire il discorso intorno al « caso italiano ».

A questi fini ci avvarremo dei dati di una ricerca comparata condotta nel 1974 e 1975 in otto paesi - Austria, Germania, Inghilterra, Olanda, Stati Uniti, piú Finlandia, Italia, Svizzera - i cui risultati sono già rilasciati per i primi cinque paesi, mentre dobbiamo alla particolare cortesia di Perti Pesonen e di Dusan Sidjanski l'autorizzazione a anticipare in questo articolo alcuni dati sulla Finlandia e sulla Svizzera ${ }^{1}$. Per ragioni di

1 La ricerca è stata coordinata da $\mathrm{S}$. H. Barnes, della Università del Michigan, al quale egualmente dobbiamo un particolare ringraziamento. Un primo volume di analisi sui primi cinque paesi, curato da $\mathrm{S}$. H. Barnes e Max Kaase (dal titolo ancora provvisorio Political Action in Western Democracies) uscirà entro il 1979. Sarà seguito da un volume curato da P. Pesonen e $\mathrm{H}$. Kerr, provvisoriamente intitolato People and their Polities, che includerà tutti gli otto paesi. Le singole indagini nazionali sono state eseguite da: L. Rosenmayr, A. Eder, I. Findl, E. Schlager (Austria); P. Pesonen, D. Matheson, R. Sankiaho (Finlandia); H. Klingemann, M. Kaase, K. Allerbeck (Germania); M. Abrams, A. Marsh (Inghilterra); G. Sartori, A. Marradi, G. Sani (Italia); P. Stoudhard, Felix Heunks (Olanda); D. Sidjanski, D. Handley, H. Kerr (Svizzera); S. H. Barnes, B. Farah, R. Inglehart, M. K. Jennings (Stati Uniti). Ogni indagine nazionale è basata su un questionario comune, al quale ogni 
spazio, e anche in ragione dei vincoli tuttora esistenti su una parte dei dati, la nostra analisi utilizzerà soltanto due variabili: le autocollocazioni dei rispettivi pubblici di massa lungo una scala sinistra-destra, e un «termometro dei sentimenti » nei confronti del clero. La prima variabile investe, potremmo dire, la dimensione ideologica (in termini sinistra-destra); la seconda la dimensione religiosa (o, se si preferisce, laico-clericale). S'intende che entrambe queste variabili verranno riferite al sistema partitico, e piú precisamente ai partiti rilevanti di ciascun sistema.

\section{Distribuzioni sul continuo destra-sinistra}

Siccome cominceremo con la variabile destra-sinistra, è bene intendersi in via preliminare su quanto, e per che cosa, «valga ». Un primo merito di questa variabile risiede nella sua comparabilità. È chiaro che in ogni paese le autocollocazioni spaziali di tipo destra-sinistra sono relative, e cioè relative al proprio spazio. Il che non toglie che destra-sinistra è ancora, tra tutte, la variabile piú « traducibile », e in questo senso meglio comparabile, tra paese e paese. Questa considerazione è confermata, come vedremo, dai nostri dati. Infatti, a dispetto della « relatività nazionale » di ogni spazio di tipo destra-sinistra, troveremo «spazi elastici », e cioè distanze diversissime, tra gli estremi destra-sinistra di ciascun paese.

Tutta diversa è la questione di quale sia l'importanza della dimensione destra-sinistra. Gran parte della letteratura nordamericana tende a darle poca importanza, perché si dà il caso che negli Stati Uniti questa dimensione ha, di fatto, un bassissimo valore discriminante, come vedremo ${ }^{2}$. $\mathrm{Ma}$ in chiave comparata

paese ha aggiunto le sue varianti. La durata media delle interviste eseguite in Italia (2.503) è stata di un'ora circa; le risposte sono state codificate in 304 variabili. La ricerca italiana è stata finanziata dal C.N.R. e da un supplemento di fondi erogati dall'Unione Industriale di Torino.

Si deve sottolineare che, stante gli accordi tra le singole equipes nazionali, $i$ valori numerici presentati in questo articolo sulla Finlandia, Italia e Svizzera, non possono essere riprodotti in altri scritti sino al 30 giugno 1980 (salvo speciale autorizzazione rispettivamente di Pesonen, Sartori e Sidjanski).

2 La letteratura americana è ben coperta e analizzata da P.E. Converse, Public Opinion and Voting Bebavior, in F. Greenstein, N. Polsby (a cura di), Handbook of Political Science, Addison-Wesley, 1975, vol. 
trovare un paese nel quale la percezione spaziale della politica conta poco, è non meno interessante di trovare un paese nel quale conta moltissimo. Anzi, è proprio questa alta variabilità che rende altamente interessante la nostra «variabile $»^{3}$. E per misurare questa varianza ci basta che la variabile in questione risulti « intelligibile », nel senso che gli interpellati non rifiutino di rispondere, diano mostra di capire di cosa si tratta, e di fatto si autocollochino sul continuo destra-sinistra. La percentuale delle risposte ottenute negli otto paesi è riportata nella Tavola 1.

Tav. 1 - Percentuali degli elettori che si collocano sul continuo destra-sinistra, per paese.

\begin{tabular}{lcc}
\hline & Percentuale & $\begin{array}{c}\text { Numero dei casi } \\
\text { nel campione }\end{array}$ \\
\hline Finlandia & 92,5 & $(1224)$ \\
Germania & 92,2 & $(2307)$ \\
Olanda & 90,3 & $(1201)$ \\
Regno Unito & 81,7 & $(1483)$ \\
Svizzera & 78,7 & $(1394)$ \\
Austria & 75,1 & $(1584)$ \\
Italia & 74,7 & $(2008)$ \\
Stati Uniti & 67,6 & $(1719)$ \\
\hline
\end{tabular}

Le percentuali della Tavola 1 trovano in coda gli Stati Uniti. Il che non è sorprendente. Semmai sorprende il fatto inedito - che nel 1974 gli americani che rifiutano la domanda siano scesi a meno di un terzo dell'elettorato. Se ne può ricavare che negli Stati Uniti assistiamo a un crescendo di «ideologizzazione ». Il che non toglie che - al paragone con gli altri

IV, spec. pp. 98-111. Vedi anche N.H. Nie, S. Verba, J. R. Petrocick, The Changing American Voter, Cambridge, Harvard University Press, 1976. Sul punto della differenza tra sinistra-destra e liberale-conservatore (il continuo che risulta piú saliente negli USA), vedi G. Sartori, Parties and Party Systems, New York, Cambridge University Press, 1976, pp. 328-35.

3 Per un approfondimento vedi R. Inglehart, H. D. Klingemann Party Identification, Ideological Preference and Left-Right Dimension among Western Mass Publics, in I. Budge, I. Crewe, D. Farlie (a cura di), Party Identification and Beyond, New York, Wiley, 1976. Il problema della validità della variabile destra-sinistra è anche esaminato da Sartori, $o p$. cit., cap. X. 
paesi - questo crescendo risulti modestissimo, come vedremo tra poco. Vediamo subito, intanto, la distribuzione delle autocollocazioni degli elettori sul continuo destra-sinistra. I profili della Figura 1 non solo facilitano i paragoni, ma si commentano da soli, o quasi.

Come si vede, le distribuzioni sono chiaramente raggruppabili in tre tipi. Un primo gruppo è dato da Stati Uniti, Germania, Inghilterra e Svizzera. Gli Stati Uniti sono il caso limite di « centralità », con la punta piú alta di tutti gli altri paesi in corrispondenza al centro del continuo destra-sinistra. Ma a parte questa differenza (che si riflette nella piú bassa sottopopolazione del versante di sinistra), i primi quattro paesi si somigliano tra loro nell'essere tutti caratterizzati da una distribuzione altamente unimodale. Segue un secondo gruppo costituito da $\mathrm{Au}-$

FIg. 1 - Distribuzione delle autocollocazioni degli elettori sul continuo destrasinistra per paese*.
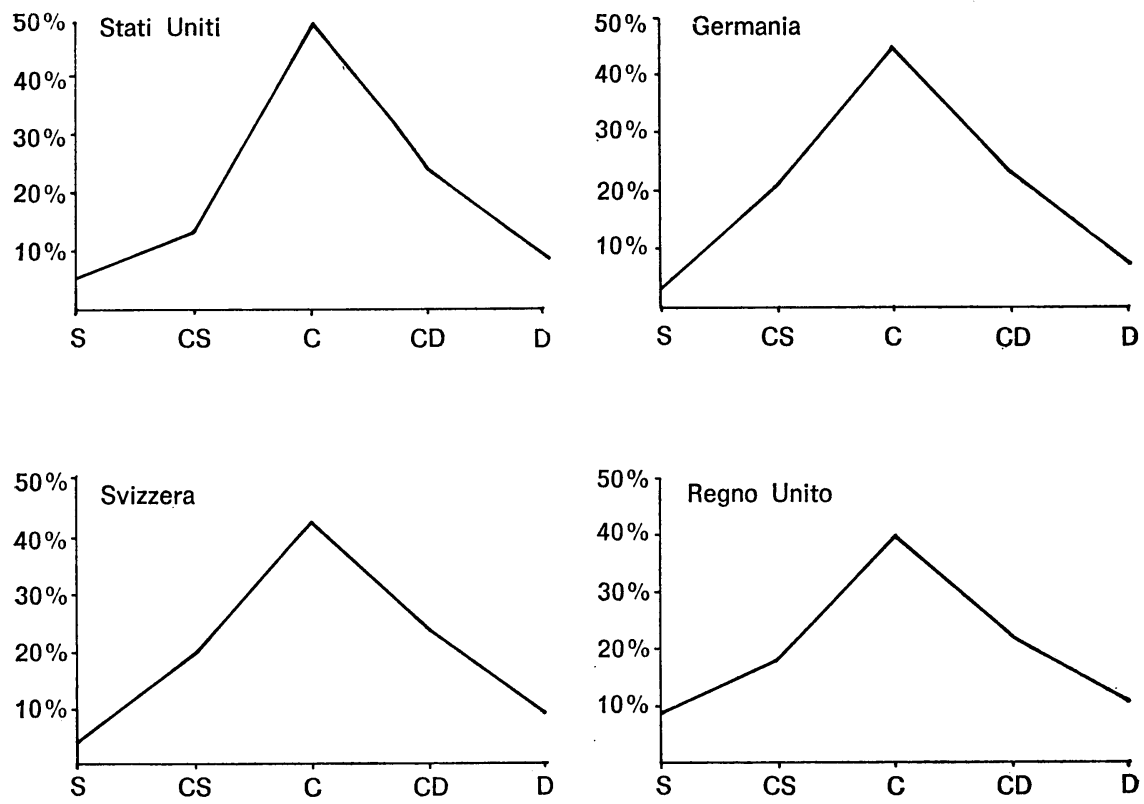

* Agli intervistati degli otto paesi è stato chiesto di autocollocarsi su una scala da 1 (estrema sinistra) a 10 (estrema destra). Per semplicità di presentazione, nella figura queste collocazioni sono state raggruppate come segue: 1-2: Sinistra; 3-4: Centro-sinistra; 5-6: Centro; 7-8: Centro-destra; 9-10: Destra. L'altezza della curva in corrispondenza di questi 5 punti indica la 
stria e Olanda. In questi due paesi la distribuzione resta unimodale, ma diventa fortemente schiacciata, o appiattita. Per quanto la moda della distribuzione sia ancora in corrispondenza con il punto centrale, tanto $\mathrm{i}$ settori di centro-destra e di centro-sinistra vi risultano molto piú popolati di quanto non lo siano nel primo gruppo di paesi. Infine, il terzo gruppo è costituito dalla Finlandia e dall'Italia, ed è fortemente atipico rispetto agli altri due tipi. La caratteristica piú saliente del terzo gruppo è una fortissima asimmetria data da una sovrappopolazione del settore che va dal centro alla estrema sinistra, alla quale corrisponde una marcatissima sottopopolazione del settore che va dal centro alla estrema destra. Si dirà che i profili della Finlandia e dell'Italia si sovrappongono meno bene dei profili dei paesi degli altri due gruppi. Infatti al segmento che va dal centro-sinistra alla sinistra corrisponde una discesa che è sensibile in Finlandia, e pressoché insensibile in Italia; laddove il

segue Fig. 1
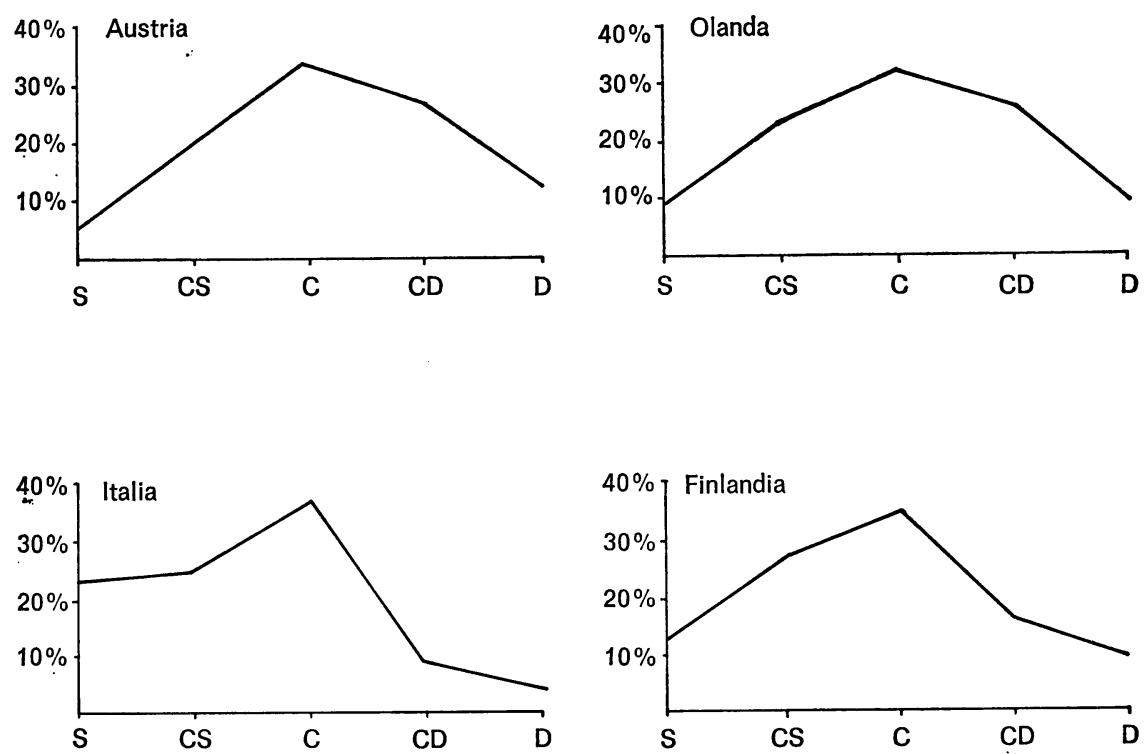

percentuale di intervistati che si sono collocati su quel particolare punto del continuo destra-sinistra. La media e lo scostamento quadratico medio, calcolati prima di raggruppare $\mathrm{i}$ dati, sono rispettivamente $\mathrm{i}$ seguenti: Stati Uniti $(5,9,1,9)$; Germania $(5,8,1,9)$; Svizzera $(5,8,2,0)$; Regno Unito $(5,7,2,3)$; Austria (6,0, 2,2); Olanda (5,6, 2,3); Italia $(4,3,2,2)$; Finlandia $(5,2,2,3)$. 
segmento che va dal centro-destra alla destra è molto piú popolato in Finlandia di quanto non lo sia in Italia. Dunque, la Finlandia è assai meno asimmetrica dell'Italia. Il che non toglie che la Finlandia risulta altamente asimmetrica quando viene paragonata agli altri sei paesi; e che una seconda moda in corrispondenza al punto di centro-sinistra della nostra scala rende la Finlandia molto simile all'Italia. Aggiungi che se disponessimo di dati comparabili sulla Francia, è lecito sospettare che un "profilo » francese collegherebbe assai bene quelli della Finlandia e dell'Italia.

Se i profili della Figura 1 vengono collegati ai sistemi di partito dei paesi in questione, e piú esattamente alla loro frammentazione (o non frammentazione) partitica, ci si avvede subito che le distribuzioni destra-sinistra non ci illuminano sul grado di frammentazione. La Svizzera è un paese a relativamente alta frammentazione partitica; eppure il suo profilo è molto simile a quelli della Germania e dell'Inghilterra, e cioè di due paesi a bassa frammentazione (2-3 partiti). Per converso, l'Austria è a tutti gli effetti un sistema a due partiti e anzi, da una decina d'anni a questa parte, il piú « puro » dei sistemi bipartitici esistenti; eppure l'aggregato delle distribuzioni destra-sinistra dell'Austria è pressoché indistinguibile da quello dell'Olan$\mathrm{da}$, che è invece un paese ad alta frammentazione partitica (con 5-6 partiti rilevanti). E giocoforza concludere, allora, che il discorso ancora non quaglia. Il che ci porta a considerare, in prima istanza, il grado di polarizzazione degli elettorati degli otto paesi.

\section{Destra-sinistra: il grado di polarizzazione}

La polarizzazione di un sistema partitico è data dalla distanza che separa (in ciascuna delle dimensioni rilevanti) le forze politiche che ne occupano i poli estremi. Quando questa distanza è riferita alla dimensione destra-sinistra, la polarizzazione in questione può essere detta ideologica. Si ricordi anche che $\mathrm{i}$ nostri dati colgono percezioni e collocazioni di tipo destra-sinistra a livello di massa. Il che equivale a dire che i nostri dati colgono la polarizzazione a livello elettorale, o di elettorato (non a livello di élites).

Come si è già avvertito, questa distanza, o questa polarizzazione, verrà qui misurata in riferimento ai partiti rilevanti: tali 
secondo le regole contabili proposte da Sartori ${ }^{4}$. Di tanto aumenta il numero dei partiti, di altrettanto è plausibile che un sistema contenga «partitini » che non hanno nessuna apprezzabile incidenza sistemica, che non modificano in alcun modo, con la loro presenza o insorgenza, la meccanica del sistema. Definiamo «irrilevante », dunque, un partito che non ba rilevanza sistemica. E il punto è che un « criterio di rilevanza » imperfetto (se tale si vuol considerare quello che adottiamo) è sempre meglio che nessun criterio. Nella fattispecie, se la distanza ideologica venisse misurata sulla base di tutti i partiti - e cioè includendo anche $i$ partiti « irrilevanti » - ne ricaveremmo una misura che non ha portato sistemico, e dunque non solo una misura inutile ma anche una misura ingannevole. Per esempio, anche in Olanda e in Austria, vi è un partito comunista; ma, appunto, un partito comunista che risulta «irrilevante », che non incide in alcun modo nel gioco competitivo o coalizionale. Se includessimo questi due «partitini » nella nostra misura di polarizzazione, l'indicazione sarebbe fuorviante, e cioè indicherebbe uno spazio competitivo che non c'è, che non è quello « reale ».

Nel caso dell'Italia il punto dibattibile è se il MSI debba essere considerato « rilevante », e come tale incluso nel computo. Al momento in cui scriviamo questa inclusione può essere messa in dubbio. Ma non era dubbia al momento al quale le rilevazioni si riferiscono, e potremmo dire fino alla elezione del 1978 del nuovo Presidente della Repubblica Pertini. Fino a quel momento, e per tutto l'arco di un ventennio, il MSI soddisfa i criteri di rilevanza: il suo voto parlamentare è stato sempre contrattato sottobanco, risultando anche determinante per una serie di atti decisivi (non ultimo dei quali l'elezione del Presidente Leone). A tutt'oggi, il 5 per cento circa dell'elettora-

4 Per i criteri di «rilevanza» di Sartori, vedi Parties and Party Systems, cit. spec. pp. 121-22 (ma anche pp. 307-15). Per detti criteri un partito può essere scontato come «irrilevante » a due condizioni: 1) quando risulta innecessario, e di fatto inutilizzato, per qualsiasi possibile coalizione, e/o 2) quando difetta di «potere di ricatto» (nel senso precisato da A. Downs in An Economic Theory of Democracy, New York, Harper \& Row, 1957). E bene precisare che la nozione di coalizione si riferisce, in prima istanza, alla coalizione di governo; ma può essere estesa anche alle coalizioni parlamentari, e cioè a accordi parlamentari con partiti il cui voto può bloccare, o sbloccare, l'attività legislativa (o che risultano decisivi per votazioni di particolare rilievo, quale l'elezione, in Italia, del Presidente della Repubblica). 
to coriaceo del MSI impedisce coalizioni di governo (maggioritarie) che non siano di centro-sinistra; il che costituisce una interferenza rilevante nella meccanica del nostro sistema politico. Sarà dunque opportuno aspettare le prossime elezioni per vedere se la secessione in atto, o altri motivi, trasformeranno il MSI in un partito irrilevante, da escludere dal conteggio. Nel qual caso - e restando immutate tutte le altre condizioni - la distanza destra-sinistra dello spettro ideologico italiano diminuirebbe. In compenso aumenterebbe, come andremo ora a spiegare, l'asimmetria o lo squilibrio, e cioè l'altra caratteristica del sistema italiano.

Ciò premesso, torniamo ai dati e vediamo quali siano in ciascun paese le forze politiche che rappresentano i poli di destra e di sinistra, e quanto grande sia la distanza che li separa. A questo fine abbiamo riportato nella Tavola 2 il valore medio delle collocazioni destra-sinistra di gruppi di elettori « contrari », isolando per ogni paese $i$ due poli, e indicando nella terza colonna la distanza tra i due. Questi dati, e la rappresentazione grafica della Figura 2 (dalla quale si possono anche desumere $i$ valori medi relativi ai simpatizzanti delle altre forze politiche), danno un quadro molto chiaro delle similarità e differenze riscontrabili in sede di polarizzazione ideologica nei nostri otto sistemi.

Come si vede, si va da un caso (gli Stati Uniti) nel quale i due poli sono vicinissimi tra loro - talché la polarizzazione destra-sinistra risulta inesistente - ai casi della Finlandia e dell'Italia, nei quali la distanza tra i poli è molto accentuata. Tra questi due estremi si collocano gli altri cinque sistemi, nei quali le distanze tra $\mathrm{i}$ due poli sono modeste. In Svizzera, Germania, Austria, Inghilterra e Olanda gli elettori dei partiti che rappresentano i poli di destra e di sinistra si differenziano (nelle rispettive collocazioni medie) assai piú di quanto non avvenga negli Stati Uniti, ma certo assai meno che in Italia e Finlandia.

La domanda, a questo punto, è: i profili della Figura 1, i «punti di taglio » che emergono dalla misura della polarizzazione (Tavola 2), e il quadro d'assieme della Figura 2, cosa ci dicono a effetto tipologico, e cioè su come classificare $\mathrm{i}$ sistemi di partito? La risposta sembra indubbia. Le nostre risultanze confermano la irrilevanza della distinzione tra bipartitismo e pluripartitismo (che rende diverso il simile), e sottolineano, per contro, l'importanza della distinzione tra pluralismo moderato 
FIG. 2 - Distanze e collocazioni dei partiti sul continuo destra-sinistra, per paese.

Austria

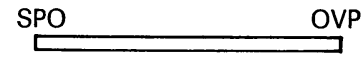

Germania

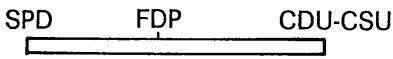

Svizzera

PS

$\mathrm{Al} \quad$ UDC PDC

$\mathrm{RD}$

Stati Uniti

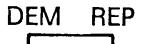

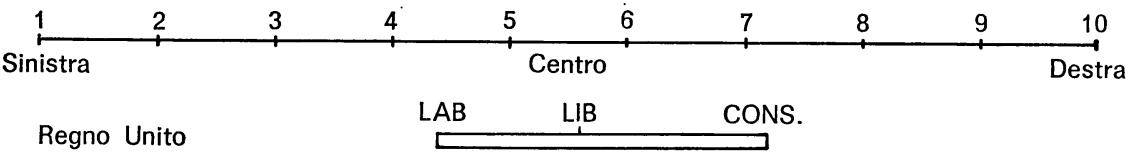

Olanda

Italia

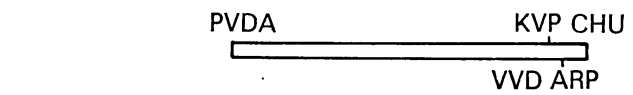

Finlandia $\frac{\text { SKDL }}{\text { SDP }}$. KES

Legenda Fig. 2

Austria: SPO, Socialisti; OVP, Popolari (Cattolici).

Finlandia: SKDL, Comunisti; SDP, Socialdemocratici; KES, Centro (Agrari); LKP, Liberali; KOK, Conservatori.

Germania: SPD, Socialdemocratici; FDP, Liberali; CDU-CSU, Democristiani.

Olanda: PvDA, Laboristi; VVD, Liberali; KVP, Cattolici; ARP, Anti-Rivoluzionari (Protestanti); CHU, Cristiano-Storici (Protestanti).

Regno Unito: LAB, Laboristi; LIB, Liberali; CONS., Conservatori.

Svizzera: PS, Socialisti; AI, Indipendenti; UDC, Agrari; RD, Radical-Democratici; PDC, Cattolici.

Stati Uniti: DEM, Democratici; REP, Repubblicani.

- fino a 4-5 partiti - e pluralismo polarizzato (che separa quel che la distinzione tradizionale confondeva) ${ }^{5}$.

I dati della nostra ricerca consentono anche di attribuire una maggiore precisione analitica alla categoria del pluralismo polarizzato. Fermo restando che la polarizzazione (ideologica) è una funzione della distanza tra i partiti estremi di un continuo

5 Per la tipologia in questione vedi, da ultimo, Parties and Party Systems, cit., capp. 5-6, e anche 9. 
Tav. 2 - Autocollocazioni medie e polarizzazione, per paese.

\begin{tabular}{llll}
\hline & Polo di sinistra & Polo di destra & $\begin{array}{r}\text { Distanza } \\
\text { (Polariz- } \\
\text { zazione) }\end{array}$ \\
\hline Stati Uniti & Democratici (5,7) & Repubblicani $(6,4)$ & 0,7 \\
Svizzera & Socialisti $(4,6)$ & Cattolici $(6,9)$ & 2,3 \\
Germania & Socialdemocratici $(4,5)$ & Democristiani $(7,0)$ & 2,5 \\
Austria & Socialisti $(4,9)$ & Popolari $(7,5)$ & 2,6 \\
Regno Unito & Laboristi (4,4) & Conservatori $(7,2)$ & 2,8 \\
Olanda & Laboristi $(4,2)$ & Protestanti CHU (7,2) & 3,0 \\
Italia & Comunisti $(2,5)$ & Neo-Fascisti $(8,2)$ & 5,7 \\
Finlandia & Comunisti (2,3) & Conservatori $(8,1)$ & 5,8 \\
\hline
\end{tabular}

destra-sinistra, e cioè tra $\mathrm{i}$ punti che definiscono lo spazio competitivo (ideologico) di un sistema partitico, il profilo dell'Italia nella Figura 1 si caratterizza per un secondo rispetto, e cioè per una marcatissima asimmetria. L'Italia ha tre mode salienti (sul centro, sul centro-sinistra e sulla sinistra) che si collocano tutte e tre sul segmento che va dal centro alla sinistra. In un precedente lavoro Sartori aveva collocato la Finlandia nel tipo del pluralismo polarizzato qualificandola, peraltro, come una fattispecie a polarizzazione controllata (e in questo senso di semi-polarizzazione). I nostri dati consentono di vedere meglio perché, ferme restando, per la Finlandia, le caratteristiche della distanza e della asimmetria, tuttavia la Finlandia (vedi sempre Fig. 1) è meno asimmetrica dell'Italia. E se asimmetria sta per « squilibrio », potremmo dire cosí: che l'Italia è un caso di pluralismo polarizzato squilibrato, mentre la Finlandia è un caso di pluralismo polarizzato piú equilibrato, o meno squilibrato.

Come è noto, alla assegnazione del caso italiano alla classe, o al tipo, del pluralismo polarizzato è stato obiettato che «manca la prova », o comunque che la polarizzazione del nostro sistema politico era tale in passato, ma non è piú tale al presente ${ }^{6}$. Per il primo rispetto, i nostri dati aggiungono una « prova ». E per il secondo rispetto i nostri dati non confortano

6 Per un dibattito d'assieme cf. F. L. Cavazza, S. Graubard (a cura di), Il caso italiano, Milano, Garzanti, 1975, vol. 1. Il dibattito è anche ripreso nella antologia curata da A. Lombardo, Il sistema disintegrato, Milano, Sugar, 1977. 
in alcun modo la asserzione che la nostra polarizzazione è in via di estinzione: in Italia (cosí come in Finlandia) le distanze destra-sinistra restano di gran lunga maggiori delle distanze che contraddistinguono $\mathrm{i}$ sistemi di pluralismo moderato. Si potrà sempre obiettare, si capisce, che la « caduta di polarizzazione » è avvenuta, in Italia, dopo le nostre rilevazioni (che risalgono alla fine del 1975) e in coincidenza con le elezioni del 1976. Resta il fatto che una successiva ricerca della Demoskopea dell'Aprile 1976 non rileva alcun trend depolarizzante, e anzi fornisce come si vede nella Figura 3 - una immagine ancor piú divaricata della nostra dei poli dello schieramento italiano ${ }^{7}$.

FIg. 3 - Struttura partitica polarizzata: Italia.

Ricerca Otto Nazioni

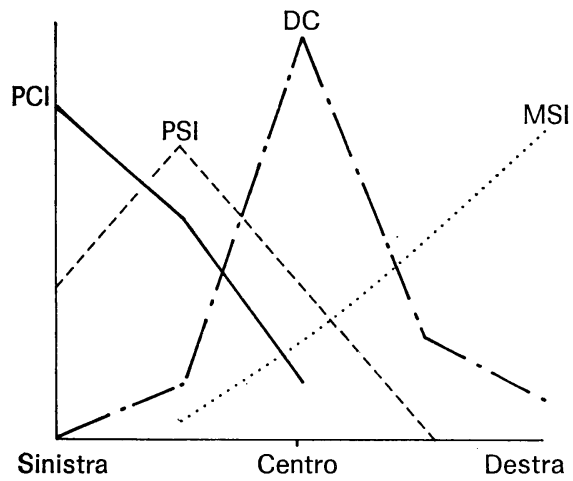

Ricerca Demoskopea

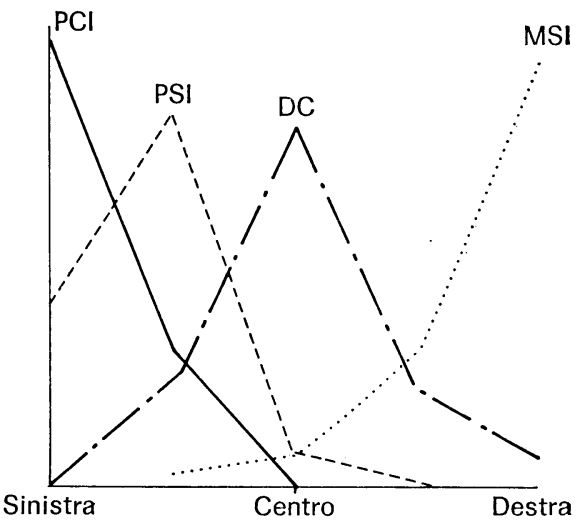

Dalla Figura 3 si ricava che in entrambe le rilevazioni le mode delle distribuzioni degli elettori PCI, DC e MSI sono nettamente differenziate e raffigurano un modello « tripolare » caratterizzato dal fatto che $\mathrm{i}$ punti intermedi (centro-destra, centro-sinistra) sono relativamente poco popolati. La differenza tra questa configurazione (che si dà, si ricordi, anche in Finlandia), e quella che caratterizza i sistemi a pluralismo moderato risulta con estrema chiarezza dal confronto tra le distribuzioni della Figura 3, e quelle della Figura 4 su Austria, Svizzera e Olanda.

7 I dati Demoskopea sono ricavati da G. Fabris, Il comportamento politico degli italiani, Milano, Angeli, 1977, Tav. 2.4, pp. 39-40. 
Fig. 4 - Strutture partitiche bipolari a bassa polarizzazione.
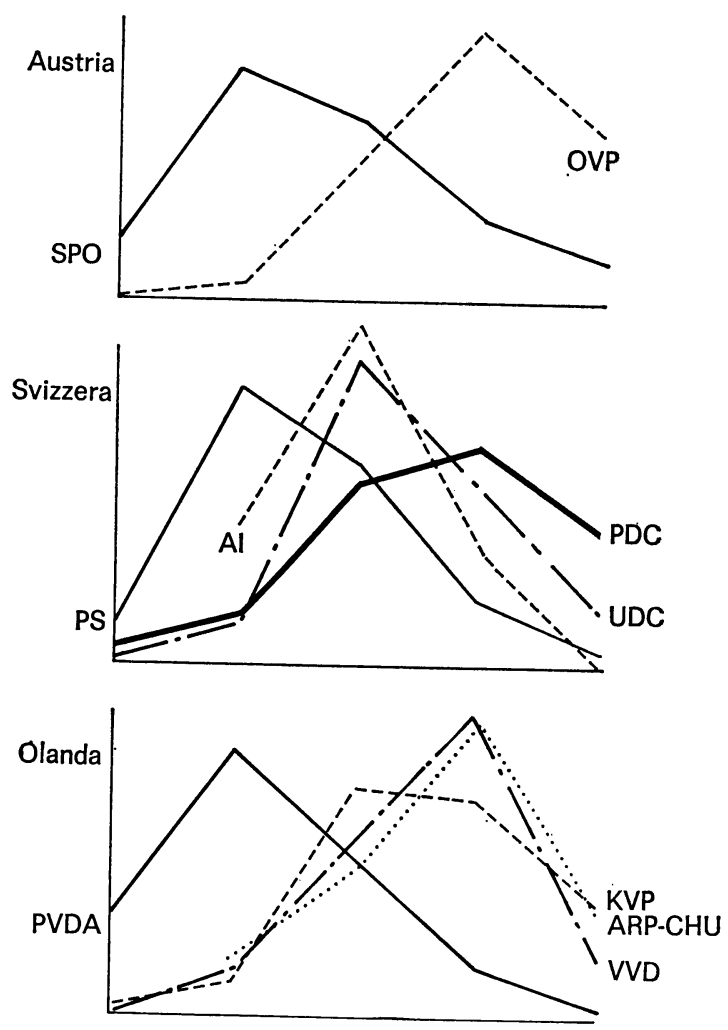

In questi ultimi sistemi (come del resto, e in maniera ancora piú accentuata, negli Stati Uniti, Germania e Inghilterra) le mode delle distribuzioni tendono a cadere sulle mezze-ali, sui punti intermedi dello spettro destra-sinistra, con la conseguenza che i poli estremi sono relativamente sottopopolati e il grado di polarizzazione nettamente inferiore.

Bobbio si chiede se, in Italia « esiste ancora un centro ${ }^{8}$. Dal punto di vista delle autocollocazioni dell'elettorato la risposta suggerita dai dati (vedi specialmente i profili nelle Figure 1 e 3) è sí: esiste ancora. Ovviamente $\mathrm{i}$ nostri dati non rispondono alla vera domanda di Bobbio, che concerne la meccanica del

8 Cf. Norberto Bobbio in "Mondoperaio" (n. 9, 1977), ora in A. Pombardo (a cura di), Le trastormazioni del comunismo italiano, Milano, Rizzoli, 1978, spec. pp. 143-47. 
sistema partitico italiano, il che non toglie che le nostre rilevazioni costituiscono un elemento da non ignorare ai fini del giudizio d'insieme.

In conclusione, chi ha ignorato e ignora, nell'interpretazione del caso italiano, il fattore " polarizzazione », ignora un fattore esplicativo di cui la ricerca sulle otto nazioni conferma - oltre ogni dubbio - la centralità. Ovviamente resta da accertare come i processi di polarizzazione e inversamente di depolarizzazione si sviluppano a livello di élite. $\mathrm{Ma}$ non lo si accerterà mai se prima non si stabilisce che la variabile da mettere in conto è, appunto, "polarizzazione ». Che è quanto abbiamo cercato di stabilire fin qui.

Torniamo ora al quadro d'insieme della Figura 2. Come si sarà già notato, i singoli partiti non si distribuiscono (nella percezione dei loro elettorati) in modo uniforme nello spazio ideologico dei rispettivi sistemi. E si sarà anche notato che in tre paesi - Svizzera, Olanda e Finlandia - la variabile destra-sinistra non discrimina a sufficienza alcuni sottogruppi di partiti. I casi di sovrapposizione, o di quasi sovrapposizione, sono soprattutto due: quello della Svizzera, dove L'UDC (il partito agrario, dei contadini o degli agricoltori) è molto vicino ai Radical-Democratici (RD), che sono a loro volta assai vicini ai Cattolici (PDC); e ancor piú l'Olanda, dove i Liberali (VVP) e i tre partiti religiosi (Cattolici, Anti-Rivoluzionari e Cristiano-Storici) fanno pressoché una ammucchiata. Dal che si ricava che almeno per questi paesi la distintività dei singoli partiti è da trovare in altre dimensioni. Ma prima di lasciare la dimensione destra-sinistra vale fare il punto, e cioè vedere sino a dove questa dimensione ci ha consentito di arrivare.

Dalla Figura 2 risulta che alla crescita del numero dei partiti corrisponde una maggiore distanza destra-sinistra in sei casi su otto: da un lato, gli Stati Uniti, la Germania, l'Austria e l'Inghilterra (due-tre partiti in corrispondenza con una piccola o media polarizzazione); e, dall'altro lato, la Finlandia e l'Italia (dove cinque-sei partiti corrispondono a una forte polarizzazione). Invece, in due casi - Svizzera e Olanda - il numero dei partiti è relativamente alto, mentre la polarizzazione è bassa, o relativamente bassa. In verità, sulla Svizzera si può discutere, e cioè questa eccezione è assai meno flagrante di quanto non sembri a prima vista ${ }^{9}$. Ma l'eccezione è flagrante nel caso

${ }^{9}$ Per varie ragioni. La maggiore è l'altissimo decentramento a livello 
dell'Olanda: 5-6 partiti con una polarizzazione appena superiore a quella dell'Inghilterra (con 2-3 partiti), e di poco superiore a quella dell'Austria (con soltanto 2 partiti).

Dunque, non è soltanto che la dimensione destra-sinistra non attribuisce una sufficiente distintività a taluni partiti. Anche la distanza tra destra e sinistra lascia quantomeno un caso, quello dell'Olanda, insoluto. È per due rispetti, allora, che dobbiamo cercare spiegazioni in altre dimensioni.

\section{La dimensione religiosa}

In astratto le altre dimensioni da considerare sono parecchie. In concreto, e cioè visti i paesi, è ovvio che nel nostro caso il primo candidato è la dimensione laico-clericale, vale a dire, in breve, la dimensione religiosa. Infatti un solo paese del nostro gruppo è polietnico, o comunque multi-linguistico: la Svizzera. Per contro, almeno cinque dei nostri paesi sono caratterizzati dalla presenza di partiti religiosi, o comunque di indubbia origine religiosa: Austria, Italia, Olanda, Germania, Svizzera. Come si è già detto all'inizio, l'indicatore che utilizzeremo per individuare la posizione dei rispondenti lungo il continuo laico-clericale è dato da un «termometro » (graduato da 0 a 100 , il cui punto neutro è 50 ) che registra i sentimenti degli intervistati nei confronti del clero.

Utilizzando le medie delle valutazioni date al clero dai diversi sottogruppi di elettori, è possibile identificare anche per questa variabile due poli significativi, che sono in questo caso quello clericale e quello laico. Le distanze tra questi due poli, cioè il grado di polarizzazione lungo la dimensione religiosa, appaiono nella Tavola 3 , in calce alla quale sono riportati anche i valori relativi agli altri sottogruppi di elettori. Per semplicità e comodità di comparazione la scala originaria è stata trasformata

cantonale. Se si considera, inoltre, che la Svizzera è un paese trilingue, o quantomeno bilingue, 4-5 partiti non costituiscono - in un contesto cosiffatto - un'alta frammentazione. A proposito della Svizzera si deve anche notare che la sua formula di governo ostacola l'applicazione dei nostri criteri di rilevanza. (La composizione del Consiglio Federale è precostituita in proporzioni prefissate, ed è limitata, dal 1959, ai quattro partiti maggiori, con esclusione degli Indipendenti). La sostanza è, comunque, che la Svizzera è un sistema a 5 partiti, ivi incluso il partito degli Indipendenti (la cui inclusione non solleva, tra l'altro, problemi, visto che non cambia in nulla la nostra misura di polarizzazione). 
TAV 3 - Polarizzazione sulla dimensione religiosa, per paese.

\begin{tabular}{lllc}
\hline & Polo clericale & Polo laico & $\begin{array}{c}\text { Distanza } \\
\text { (Polariz- } \\
\text { zazione) }\end{array}$ \\
\hline Stati Uniti & Repubblicani $(8,4)$ & Democratici $(8,2)$ & 0,2 \\
Regno Unito & Conservatori $(6,7)$ & Laboristi $(6,4)$ & 0,3 \\
Austria & Popolari $(8,2)$ & Socialisti $(6,1)$ & 2,1 \\
Germania & Democristiani $(6,6)$ & Socialisti $(4,4)$ & 2,2 \\
Svizzera & Cattolici $(8,5)$ & Socialisti $(5,5)$ & 3,0 \\
Finlandia & Centro-KES (8,1) & Comunisti (5,0) & 3,1 \\
Olanda & Protestanti ARP (8,4) & Laboristi-PvDA (5,2) & 3,2 \\
Italia & Democristiani (7,5) & Comunisti $(3,3)$ & 4,2 \\
\hline
\end{tabular}

I valori relativi agli altri sottogruppi di elettori sono:

Regno Unito: Liberali 6,7

Germania: Liberali 4,6

Svizzera: $\quad$ Agrari - UDC 5,9; Indipendenti 5,4; Radical Democratici 5,7

Finlandia: $\quad$ Socialisti 6,4; Liberali LKP 6,6; Conservatori 7,8

Olanda: $\quad$ Cattolici 7,9; Liberali 4,2; Protestanti-CHU 8,0

Italia: $\quad$ PSI 4,6; PSDI-PRI 5,1; PLI 4,3; MSI 4,6

in una scala da 1 a 10 analoga a quella usata per la dimensione destra-sinistra.

La prima osservazione è che negli Stati Uniti e in Inghilterra la dimensione religiosa non ha alcuna rilevanza sulle preferenze partitiche (a tal punto che la classificazione non ha, qui, senso), laddove nei restanti sei paesi esiste una associazione tra atteggiamento nei confronti del clero e scelte partitiche. All'interno dei sei paesi in questione le differenze sono peraltro significative. Dopo tutto il gran parlare che si è fatto, per l'Austria, di Lager, di socialisti e cattolici come di due mondi chiusi e recintati, sorprende vedere che i socialisti austriaci vedano il clero con favore (sono sopra a 50 ), e ancor piú sorprende la grandissima differenza tra socialisti austriaci $(6,1)$ e tedeschi $(4,4)$. In effetti, $i$ socialisti tedeschi sono, tra tutti i socialisti degli otto paesi, i meno favorevoli al clero, i piú anticlericali: anche questo un fatto sorprendente. Sull'altro fronte, i piú clericali di tutti (quantomeno per l'indicatore «percezione favorevole del clero ») sono i cattolici del PDC svizzero $(8,5)$, seguiti dagli austriaci $(8,2)$, dal Centro o Agrari finlandesi $(8,1)$. I nostri «vicini » alla DC sono alquanto piú tiepidi: stanno al penultimo posto in graduatoria con 7,5 (un 
campanello di allarme per le élites integraliste della DC), seguiti solo dalla CDU-CSU tedesca con 6,6 (il che conferma la forte deconfessionalizzazione di quel partito democristiano).

Parlando in generale, con riferimento alla misura di polarizzazione religiosa vediamo che gli otto paesi cadono in quattro classi: i) Stati Uniti e Inghilterra, dove la dimensione religiosa è del tutto irrilevante in sede di affiliazioni partitiche; ii) Austria e Germania, con modesta polarizzazione (seppur risultante da combinazioni molto diverse; la relativamente forte laicità dei socialisti tedeschi è compensata dalla deconfessionalizzazione dei democristiani, laddove la forte cattolicità dell'SPÖ Austriaco è compensata dal nessun anticlericalismo dell'ÖVP); iii) Svizzera, Finlandia e Olanda, vicinissimi tra loro con una polarizzazione religiosa che potremmo dire media (che va da 3 a 3,2 punti); iv) l'Italia, con forte (comparativamente o relativamente forte) polarizzazione religiosa. La novità piú interessante di questi risultati è dunque che lungo la dimensione laico-confessionale l'Italia si stacca molto nettamente dagli altri paesi, anche dalla Finlandia (alla quale l'Italia era molto simile lungo la prima dimensione). E non solo la nostra misura della polarizzazione religiosa scorpora la Finlandia dall'Italia, ma piazza l'Olanda come il caso piú prossimo all'Italia. Quest'ultimo accostamento non è significativo se ci limitiamo ai valori della misura, ma diventa significativo se guardiamo alle singole gradazioni del termometro. Gli Anti-Rivoluzionari, e cioè uno dei due partiti protestanti dell'Olanda, sono fortemente religiosi $(8,4)$; e tutti $\mathrm{i}$ valori dei tre partiti religiosi dell'Olanda sono piú alti del valore della DC italiana $(7,5)$. Pertanto lo scarto italiano è dovuto al bassissimo valore $(3,3)$ del PCI - davvero un valore da qualificare « ostilità anticlericale » — che è il piú basso, nel nostro termometro, per tutti i partizzanti di tutti i paesi.

Vediamo ora che cosa la nostra seconda dimensione aggiunga alla prima. Una prima domanda è: la dimensione laico-clericale discrimina quel che la dimensione destra-sinistra non riusciva a discriminare? Per rispondere vediamo quale sia la collocazione dei diversi sottogruppi di elettori nello spazio creato dalla intersezione delle due dimensioni. Le distribuzioni della Figura 5 ci dicono che la risposta è sí.

In Svizzera la nostra dimensione separa nettamente i Cattolici (PDC) dall'UDC (Indipendenti) e dai Radical-Democratici (RD). In Olanda separa nettamente i Liberali (VVD) dai tre 
FIG. 5 - Collocazione dei partiti nello spazio bidimensionale ideologia-religione.
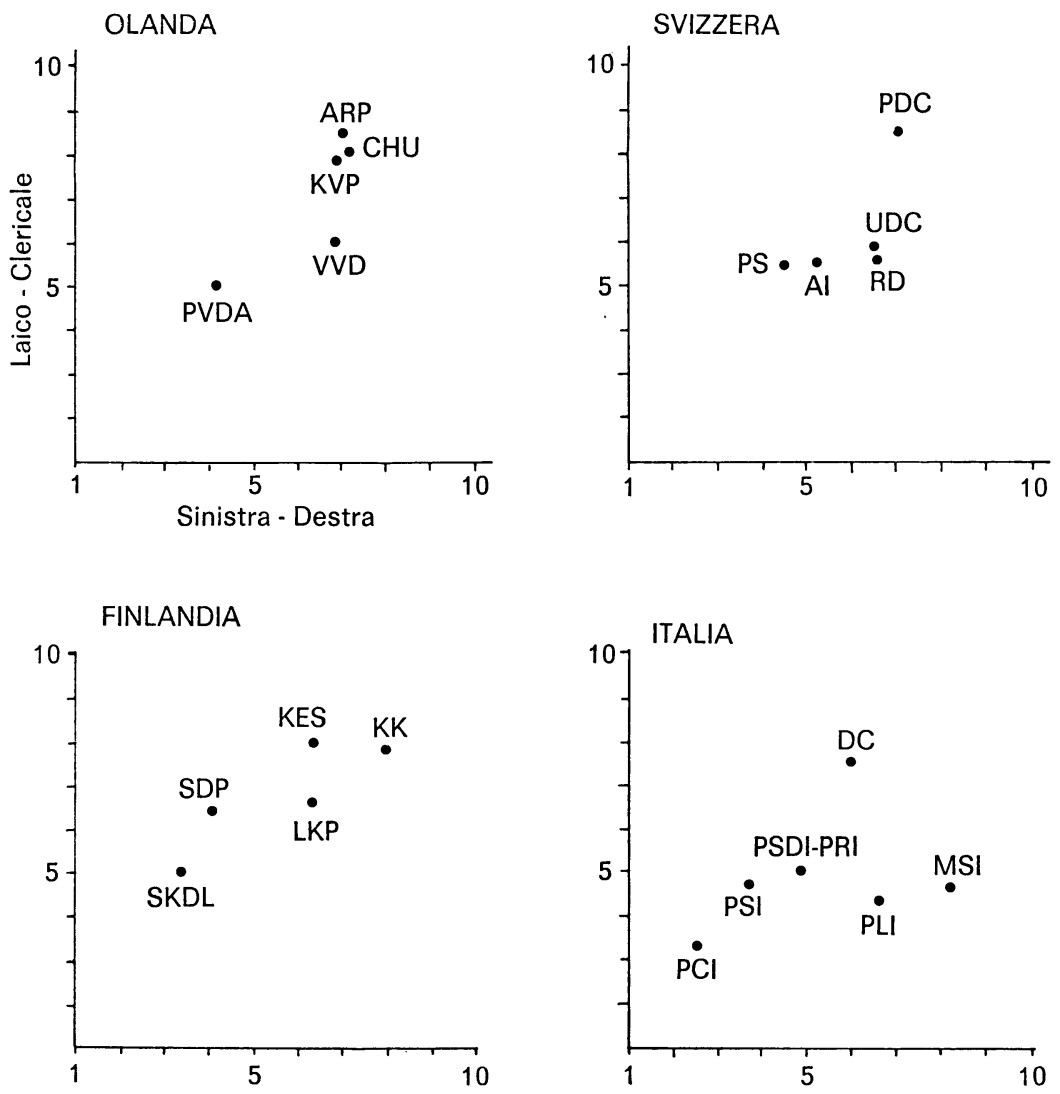

partiti religiosi. Anche in altri paesi la dimensione religiosa seppur meno indispensabile - aggiunge valore discriminante. In Finlandia serve a separare il Centro (Agrari) e l'LKP (molto vicini nella dimensione destra-sinistra). Anche in Italia la dimensione laico-clericale attribuisce una maggiore distintività, o se si vuole uno spazio proprio, ai tre partiti minori che non a torto (ci dicono le nostre misure) vengono chiamati « laici ». Pertanto la nostra dimensione manca il segno solo nel non differenziare i tre partiti religiosi dell'Olanda, e specie i cattolici $(7,9)$ dai protestanti del CHU $(8,0)$. Ma è chiaro che la nostra misura non può discriminare tra partiti religiosi (a meno che non differiscano sensibilmente in religiosità). E dunque 
questa eccezione non ci turba: ci dice soltanto che se vogliamo discriminare tra cattolici e protestanti ci occorre un indicatore diverso.

Una seconda domanda è: che rapporto sussiste tre le due dimensioni nei nostri otto sistemi? Il quesito è importante perché una forte correlazione tra le due scale indicherebbe la esistenza di cleavages coincidenti, che si sommano o cumulano. Nella fattispecie il cleavage ideologico rafforzerebbe quello religioso, e viceversa. Per contro, l'assenza di correlazione indicherebbe che le dimensioni sono indipendenti e avremo dunque il caso di cross-cutting cleavages, di linee di divisione che non si cumulano ma si incrociano senza, quindi, alcun effetto di rinforzo.

I coefficienti di correlazione della Tavola 4 indicano che in nessuno degli otto paesi i cleavages sono del tipo coincidente o rinforzante: sono tutti incrocianti. Ma nell'ambito di questa seconda configurazione le variazioni sono notevoli. In Inghilterra e negli Stati Uniti l'incrociamento è quasi perfetto, quasi ad angolo retto. Man mano che scendiamo lungo la tavola l'angolo si restringe, e i due paesi che piú si avvicinano a una struttura di cleavage di tipo coincidente e rinforzante (piú prossimi a due linee di divisione parallele) sono l'Olanda e l'Italia. Nella misura in cui i conflitti politici dipendono dalla struttura dei cleavages, l'Olanda e l'Italia hanno il piú alto potenziale di conflittualità (rispetto, s'intende, alle due variabili in esame).

La Tavola 4 può anche essere letta come una misurazione che afferisce alla teoria della democrazia consociativa. Come è noto, questa teoria si applica alle «società segmentate ${ }^{10}$, e cioè caratterizzate da linee di divisione rinforzanti, tali, appunto, da « segmentarle », da spezzarle in sotto-comunità internamente compatte, ma esternamente isolate l'una dall'altra. I casi tipici, e anzi tali per antonomasia, di società segmentate sussunte nel modello consociativo sono tutti presenti nella nostra indagine: sono l'Olanda, la Svizzera e l'Austria. Purtroppo, come ognuno può vedere, vanno in ordine sparso: la Svizzera è

10 E la dizione di H. Daalder, The Netherlands: Opposition in a Segmented Society, in R. A. Dahl (a cura di), Political Oppositions in Western Democracies, New Haven, Yale University Press, 1966; e di Val Lorwin, Segmented Pluralism: Ideological Cleavages and Political Cobesion in the Smaller European Democracies, in "Comparative Politics », gennaio 1971. Lijphart dice, nel suo ultimo volume, «plurale » (Democracy in Plural Societies, New Haven, Yale University Press, 1977); ma l'etichetta ci sembra meno azzeccata. 
TAv. 4 - Correlazioni tra variabile ideologica e variabile religiosa, per paese.

\begin{tabular}{lcc}
\hline & Coefficienti & N. \\
\hline Regno Unito & 09 & $(1190)$ \\
Stati Uniti & 10 & $(1152)$ \\
Svizzera & 20 & $(1041)$ \\
Germania & 27 & $(2113)$ \\
Austria & 28 & $(1183)$ \\
Finlandia & 33 & $(1126)$ \\
Italia & 40 & $(1469)$ \\
Olanda & 41 & $(1072)$ \\
\hline
\end{tabular}

assai piú vicina al cross-cutting degli Stati Uniti che non a quello dell'Olanda; l'Austria è pressoché identica, nel suo angolo di cleavage, alla Germania. Detto in breve: la «classe » delle società segmentate non è una classe. Con il che viene anche a cadere un retroterra della teoria del consociazionalismo. Viene a cadere, s'intende, rispetto alla correlazione tra ideologia e religione. Può darsi che la democrazia consociativa possa essere ripristinata « come classe » da altre variabili, per esempio correlando classe e religione (o anche classe, lingua e religione). Può ben darsi, cioè, che l'angolo di cleavage si restringa e risulti simile - per Svizzera, Austria e Olanda - quando le correlazioni fanno capo a misure di classe. Può darsi; ma intanto $\mathrm{i}$ nostri dati suggeriscono che le premesse sociologiche della teoria della democrazia consociativa sono da rivedere.

La posizione dei diversi gruppi di elettori nelle due dimensioni interessa anche per un terzo aspetto, che è poi di notevole rilievo. Abbiamo visto quali siano le distanze che separano in ciascun paese i poli delle due dimensioni prese separatamente. $\mathrm{Ci}$ dobbiamo ora chiedere qual è la distanza complessiva e qual è, cioè, il grado di polarizzazione congiunta che caratterizza i nostri sistemi? La risposta al quesito è fornita dal grafico della Figura 6. In essa il segmento tracciato in corrispondenza a ciascun paese rappresenta la distanza globale data dalla somma delle distanze misurate sulle due scale. Le due porzioni di ciascun segmento corrispondono rispettivamente alla distanza sulla dimensione destra-sinistra (parte chiara), e sulla dimensione religiosa (parte scura). Abbiamo cosí non solo un indice del grado complessivo di polarizzazione, ma anche una misura sia pure approssimativa della relativa importanza delle due. $\mathrm{Ne}$ 
risulta non solo una polarizzazione complessiva che vede in testa l'Italia e all'ultimo posto gli Stati Uniti, ma che i sistemi a frammentazione pronunciata sono anche quelli con una polarizzazione piú forte. Se non fosse per il caso della Svizzera, la corrispondenza sarebbe piena. Merita anche sottolineare il netto distacco tra Italia e Finlandia da un lato, e tutti gli altri paesi dall'altro. Il grado di polarizzazione in Italia e Finlandia è dieci volte piú alto di quello degli Stati Uniti, tre volte piú alto di quello dell'Inghilterra, e due volte superiore a quello dell'Austria e della Germania.

Per chiarire ulteriormente il portato di questi dati soprattutto per quanto concerne l'Italia, è utile fissare l'attenzione sulla distanza che separa alcuni sottogruppi significativi di elettori italiani rispetto a quelli di altri paesi, come nella Tavola 5 .

E significativo, ad esempio, il contrasto tra la coppia CDU-SPD in Germania (o quello OVP-SPO in Austria), e la coppia DC-PCI in Italia. Risulta che quest'ultima distanza è di ben 7,6 punti, mentre quella relativa alle prime due coppie è di 4,7. Lo stesso dicasi per il confronto tra la distanza che intercorre tra i simpatizzanti dei nostri due maggiori partiti e altre coppie significative. La distanza tra laboristi (PvDA) e cattolici (KVP) in Olanda è di 5,4 punti, quella tra Socialisti e Cattolici in Svizzera è 5,3; per non dire della distanza tra laboristi e conservatori in Inghilterra, che è ancora piú bassa $(3,1)$ e corrisponde a meno della metà della distanza tra PCI e DC. L'unico paese in cui si riscontrano distanze paragonabili all'Italia è la Finlandia, dove troviamo una differenza di 7,1 per la coppia Comunisti-Centro, e di 8,6 tra Comunisti e Conservato-

TAv. 5 - Distanze tra alcune coppie significative di partiti.

\begin{tabular}{lccc}
\hline & \multicolumn{2}{c}{ Dimensioni } & \\
& Destra-sinistra & Laico-clericale & Totale \\
\hline DC-PCI (Italia) & 3,4 & 4,2 & 7,6 \\
CDU-SPD (Germania) & 2,5 & 2,2 & 4,7 \\
OVP-SPO (Austria) & 2,6 & 2,1 & 4,7 \\
PvDA-KVP (Olanda) & 2,7 & 2,7 & 5,4 \\
PDC-PS (Svizzera) & 2,3 & 3,0 & 5,3 \\
Cons.-Lab. (R. Unito) & 2,8 & 0,3 & 3,1 \\
KES-SKDL (Finlandia) & 4,0 & 3,1 & 7,1 \\
KK-SKDL (Finlandia) & 5,8 & 2,8 & 8,6 \\
DC-PSI (Italia) & 2,2 & 2,9 & 5,2 \\
\hline
\end{tabular}


FIG. 6 - Distanze complessive ideologico-religiose, per paesi.

Stati Uniti $(0,9)$

Regno Unito $(3,1)$

Austria $(4,7)$

Germania $(4,7)$

Svizzera $(5,3)$

Olanda $(6,2)$

Finlandia $(8,9)$
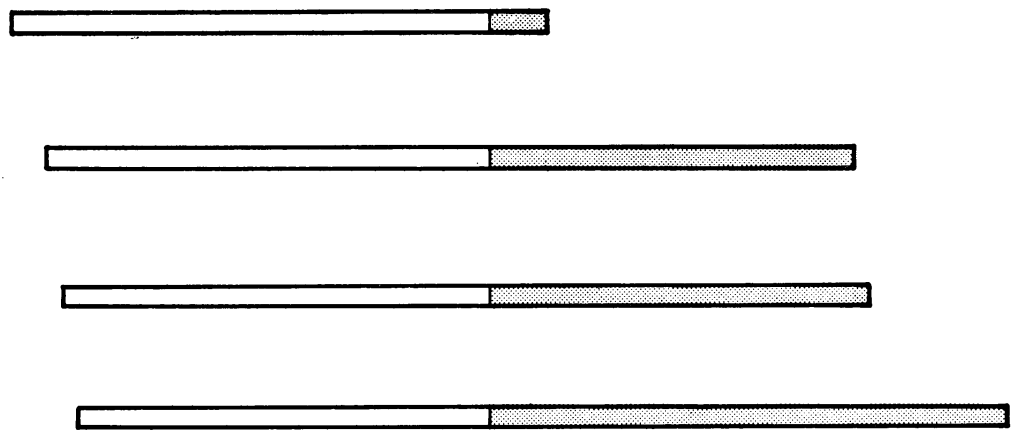

Italia $(9,9)$ 
ri. Non occorre insistere oltre sulle similarità tra Italia e Finlandia e sulle differenze tra questi sistemi di pluralismo polarizzato e quelli di pluralismo moderato. Basterà notare che nel sistema italiano la distanza assimilabile a quelle che si danno nei paesi a pluralismo moderato è quella tra DC e PSI.

\section{Democrazie facili e difficili}

È giunto il momento di chiedersi come questi dati aiutino a capire perché alcune democrazie funzionino, o funzionino meglio di altre, che non funzionano. Una dottrina semplicistica e oramai datata - di cui la dottrina del « bipartitismo imperfetto » è una sottospecie - asserisce che $\mathrm{i}$ sistemi bipartitici facilitano il funzionamento di un sistema democratico, mentre quelli pluripartitici lo renderebbero difficile. Questa spiegazione, che fa della variabile «frammentazione del sistema partitico » il fattore chiave, è già stata abbondantemente criticata; e il suo maggiore difetto è che il criterio della frammentazione non consente di separare i sistemi multipartitici nei quali la democrazia è relativamente facile, da quelli in cui è difficile.

La spiegazione che proponiamo - confortata dai nostri dati - è che il grado di frammentazione non è l'unica variabile importante, e neppure quella decisiva. Ad essa vanno aggiunte il grado di polarizzazione del sistema, e la natura cumulativa, o meno, dei suoi cleavages. La frammentazione richiede, di regola, governi di coalizione: e la meccanica della creazione e mantenimento delle coalizioni è certamente un fattore che può incidere in maniera negativa sul funzionamento del sistema. $\mathrm{Ma} \mathrm{i}$ problemi ovviamente si moltiplicano e aggravano quando le coalizioni non sono omogenee ma scollate, e cioè quando sono coalizioni tra soci distanti l'uno dall'altro. Il che equivale a dire che il funzionamento delle coalizioni dipende dalla polarizzazione. Tanto maggiore è la polarizzazione, tanto maggiore è la probabilità di coalizioni i cui soci si paralizzano a vicenda, di coalizioni inerti o altrimenti sussultorie. Le difficoltà aumentano ancora - e qui entra in gioco la nostra terza variabile quando alla polarizzazione si aggiunge una struttura di cleavages di tipo cumulativo o rinforzante.

I dati qui presentati valgono dunque a precisare in quale, $o$ quali, dei nostri sistemi politici esistano condizioni che ne facilitano, o inversamente ostacolano, il funzionamento. Tali condi- 
zioni sono riassunte nel prospetto della Tavola 6 , nella quale il segno meno indica l'assenza di una determinata caratteristica, e i piú (da uno a quattro) indicano « impressionisticamente » il grado o intensità della sua presenza.

TAv. 6 - Condizioni di funzionalità in otto democrazie.

\begin{tabular}{lccc}
\hline & Frammentazione & Polarizzazione & $\begin{array}{c}\text { Cleavages } \\
\text { cumulativi }\end{array}$ \\
\hline Stati Uniti & - & - & - \\
Regno Unito & + & + & - \\
Austria & - & ++ & + \\
Germania & + & ++ & + \\
Svizzera & +++ & ++ & + \\
Olanda & +++ & ++++ & ++ \\
Finlandia & +++ & +++++ & + \\
Italia & +++ & & ++ \\
\hline
\end{tabular}

Dal prospetto si vede nei nei primi quattro paesi la bassa frammentazione, una bassa o moderata polarizzazione, e una struttura di cleavages poco cumulativa, convergono nel costituire condizioni pressoché ottimali (rispetto, naturalmente, ai fattori considerati, che non sono certo gli unici); mentre il sistema italiano, con la sua marcata frammentazione, con la sua forte polarizzazione, e con cleavages che si cumulano almeno in parte, si colloca all'altro estremo.

Beninteso, i nostri dati registrano situazioni esistenti a livello di massa, mentre le coalizioni sono gestite dalle élites. Ciò ricordato, resta vero che le élites non operano in un vuoto e che sono quantomeno condizionate dalla polarizzazione a livello di massa. Non si può escludere, con questo, che la cura consociativa risulti applicabile anche ai sistemi di pluralismo polarizzato. Finora, tuttavia, questo rimedio ha funzionato solo nei sistemi caratterizzati da una polarizzazione moderata. Certo, comportamenti convergenti delle élites possono ridurre la polarizzazione a livello elettorale. Ma questa è una operazione che richiede buona fede, fiducia, e tempi lunghi ${ }^{11}$.

11 Le rilevazioni longitudinali di G. Sani lungo la dimensione destra-sinistra indicano, difatti, variazioni temporali estremamente lente, o vischiose. Vedile nel vol. coll. curato da $\mathrm{H}$. R. Penniman, Italy at the Polls: The Parliamentary Election of 1976, Washington, American Enterprise Institute, 1977, p. 101, Tavv. 3-6. 